\title{
A model for the chemistry of defects in bakelite plates exposed to high-radiation environment
}

\author{
T. Greci ${ }^{1}$, F. Felli, G. Saviano \\ Sapienza Universita`di Roma and Laboratori Nazionali di Frascati dell'INFN \\ E-mail: tom.greciegmail.com \\ L. Benussi, L. Passamonti, D. Piccolo, D. Pierluigi, A. Russo \\ Laboratori Nazionali di Frascati dell'INFN
}

\begin{abstract}
Results of a study on the formation of defects produced on the inner surface of the bakelite electrodes of RPC detectors are described. A mechanism for the formation of these defects is proposed.
\end{abstract}

XI workshop on Resistive Plate Chambers and Related Detectors (RPC2012)

INFN-Laboratori Nazionali di Frascati, Italy

February 5-10, 2012 


\section{Introduction}

Resistive Plate Counters (RPC) [1] are used extensively in muon detectors of several experiments at the Large Hadron Collider (LHC) of CERN, Geneva (Switzerland). RPCs use fluorine-based gas mixtures whose main component is Freon. Due to the high reactivity of fluorine-based gas mixture used, it is very important to study the material compatibilities and all radiation-damage related issues.

Defects on the inner surface of lineseed oil-coated bakelite plates have been observed in the past as a consequence of exposure to high-radiation enviroment[2][3][4][5][6]. While the effect of the increase in dark current and eventually discharge is agreed upon, little is known on the formation mechanism of defects.

A systematic investigation has been proposed [7][8][9] aimed to clarify the interaction of gas mixture with materials used in the RPC gas recirculation system of experiments at LHC such as the Compact Muon Solenoid (CMS) experiment [10]. The study is structured in three phases. Gas mixture purifiers are studied with cosmic rays. Furthermore, analyses on bakelite composition are carried out along with studies on interaction of gas mixture, filters and bakelite defects due to their interaction.

This paper reports on preliminary results of defects in bakelite plates exposed to high radiation doses. A model of the generation of the defects on the inner surface of the gaps is proposed.

\section{Setup and measurements}

RPC gaps from the CMS experiment at the CERN LHC are exposed to radiation at the CERN Gamma Irradiation Facility (GIF) where a $650 \mathrm{GBq}$-intensity, ${ }^{137} \mathrm{Cs}$ source is used. Typical gap dimension is $(1 \times 2) \mathrm{m}^{2}$, while the gas mixture used is $95.2 \% \mathrm{C}_{2} \mathrm{H}_{2} \mathrm{~F}_{4}-4.5 \%$ Iso$\mathrm{C}_{4} \mathrm{H}_{10}-0.3 \% \mathrm{SF}_{6}$ humidified at about $40 \%$. Gaps are operated at a $9 \mathrm{kV}$ high voltage supply typically. Details of exposures are reported elsewhere [11][12].

Gaps are composed of 2-mm-thick bakelite planes with a linseed oil coating enclosing a 2-mm gas volume. Bakelite is a plastic laminate obtained by the soaking of wetted paper sheets in several types of resins. A catalyst agent (activator) is used, generally $\mathrm{NaOH}$. Plastic laminates are produced by using mainly melaminic and fenolic resins. For the RPC gaps, a laminate [13] [14] composed of a bulk of paper soaked in fenolic resin was used, surrounded by two outer layers of paper soaked in melaminic resin, up to a $2 \mathrm{~mm}$ thickness [15]. In CMS RPC detectors, the inner layer is coated with linseed oil [16]. 
The effect of gas mixture on bakelite are known, in particular defects have been observed on the inner surface of gaps after operation in closed loop mode under high radiation doses. Defects have been previously identified as $\mathrm{NaF}$ structures [9][17].

In this study, visual inspection of all inner surfaces of bakelite gaps has revealed the presence of defects (Fig.1,2)

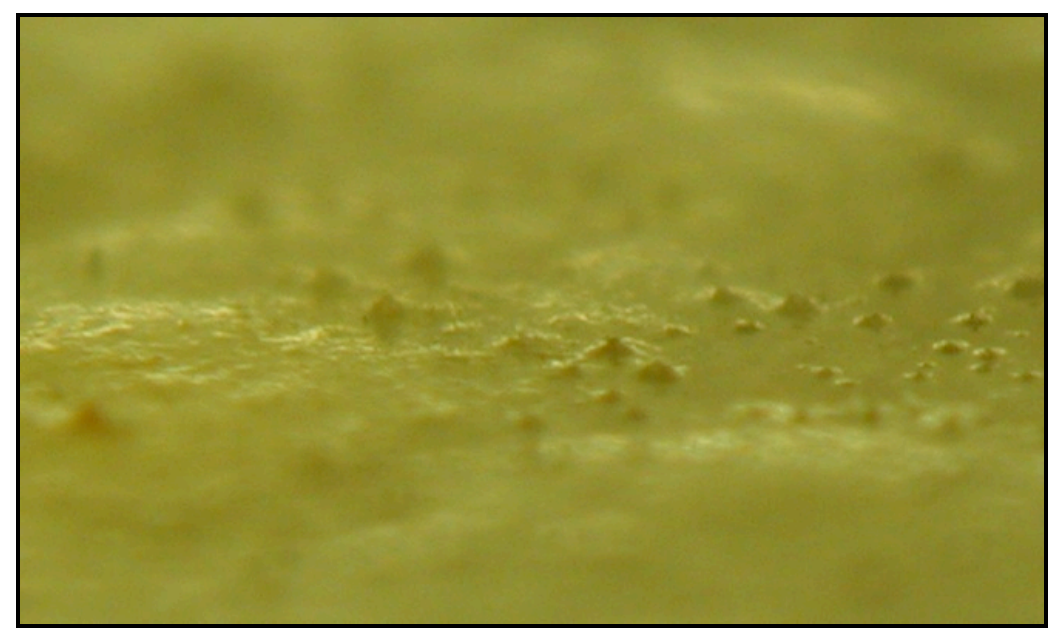

Figure 1 Bakelite surface defects on optical microscope (60x)

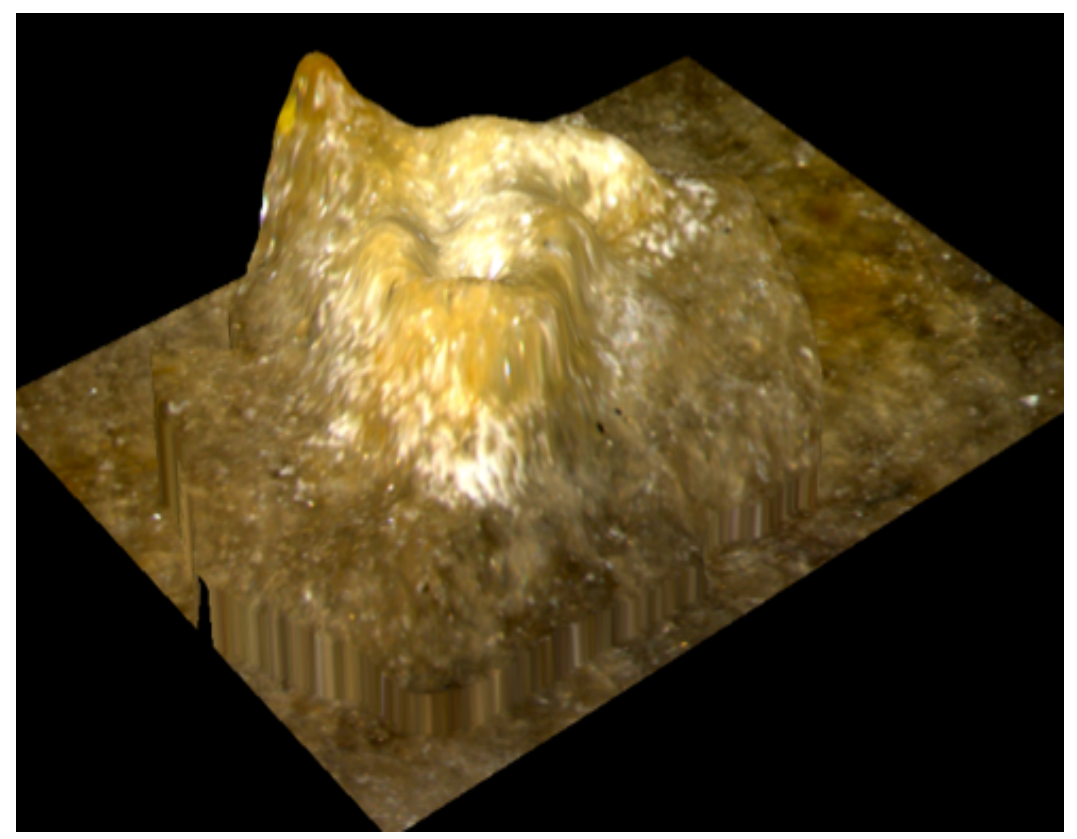

Figure 2 3D reconstruction of a typical defect (200x) 
All gaps show a heterogeneous surface characterized by cracks and discontinuities as show in Fig.3.

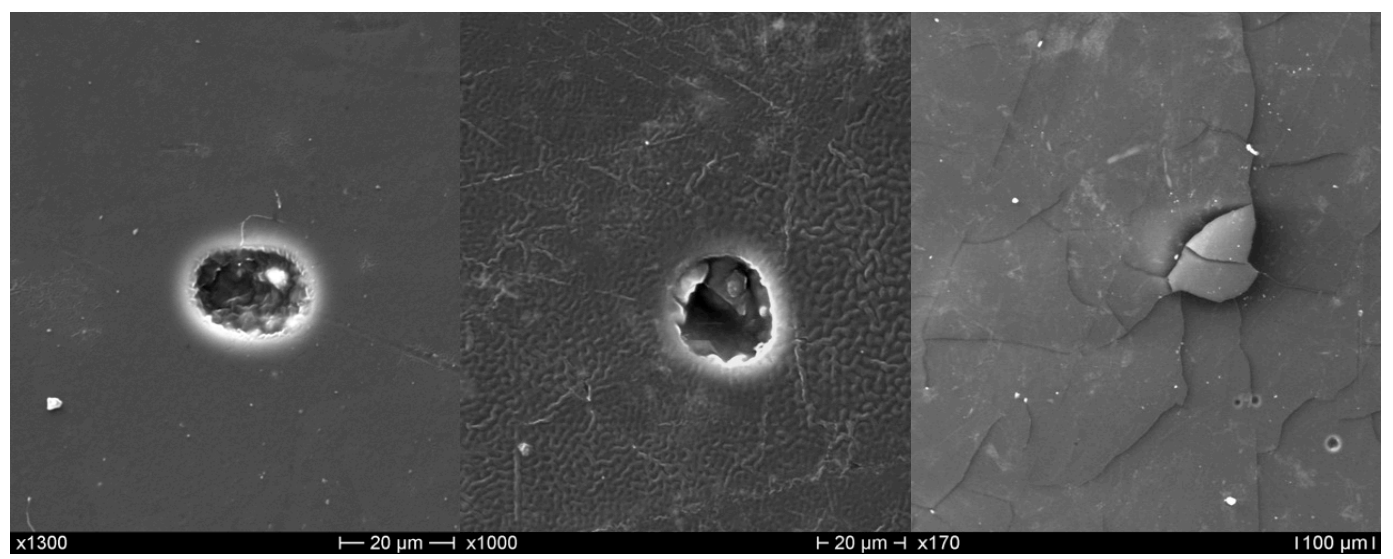

Figure 3 Surface of bakelite as seen in Scanning Electron Microscope (SEM) analysis

Following visual inspection, five gaps were mapped and the geometrical distribution of the most evident defects recorded (Fig.4). A clear correlation with gas inlet and a possible correlation with gas outlet is observed.

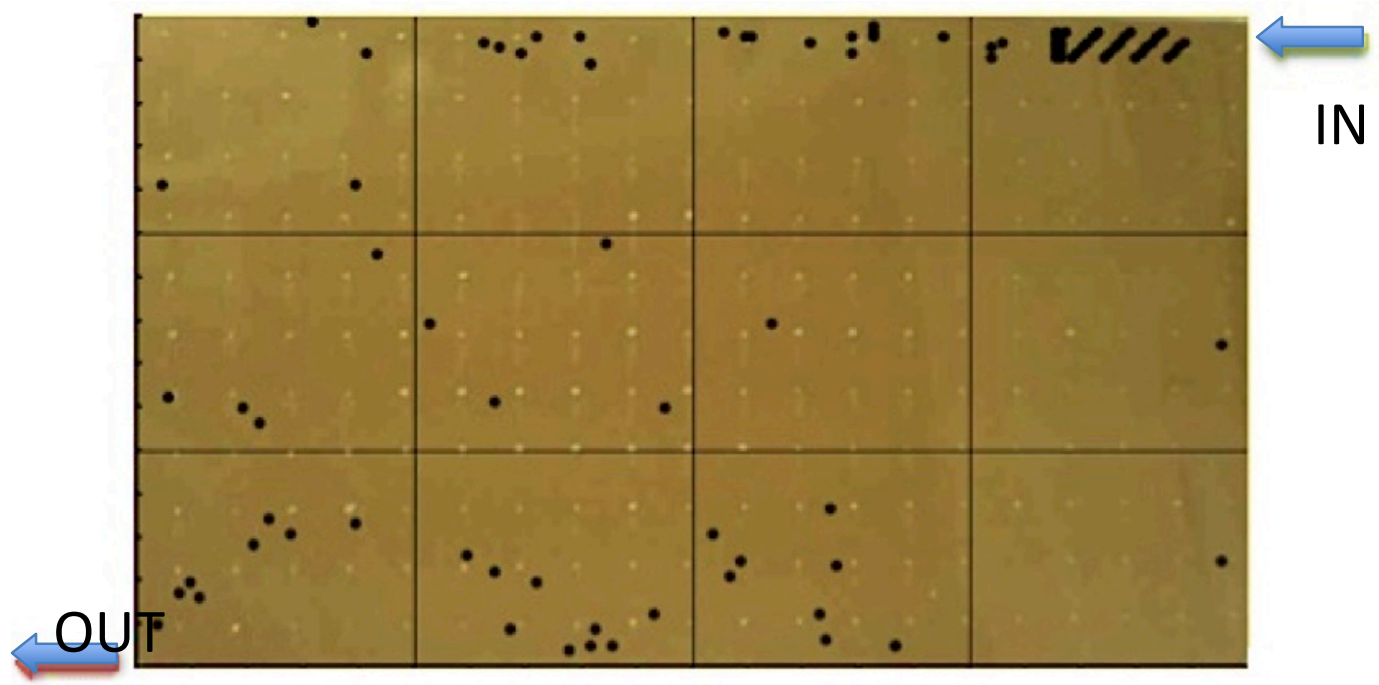

Figure 4 Mapping of defects in a $(1 \times 2) \mathrm{m}^{2}$ gap, showing gas inlet and outlet

Water content was studied by measuring the absorption time of a $(2.0 \times 2.4) \mathrm{cm}^{2}$ sample. The water absorbed was found 0.047 litres in 4 hours (Fig.5). 


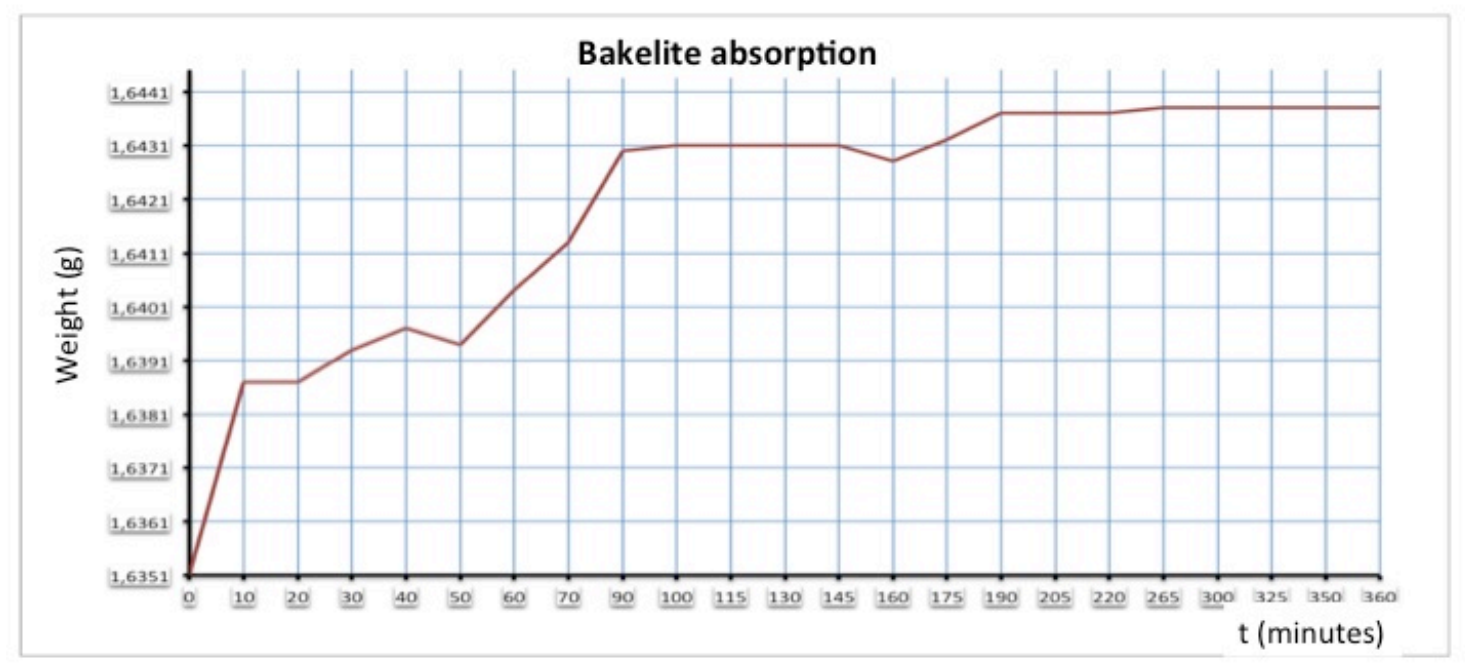

Figure 5 Bakelite absorption of water

An x-ray diffraction / scanning electron microscope (XRD/SEM) analysis was performed on twenty defected samples and twelve undefected samples. All twenty defected samples show presence of fluorine and sodium. No sodium is observed in the undefected samples. Figure 6 shows a typical XRD/SEM spectrum of defect. The fractions of $\mathrm{F}$ and $\mathrm{Na}$ components in twenty defects is listed in Table 1. The average content measured by this study is shown in Table 2. 


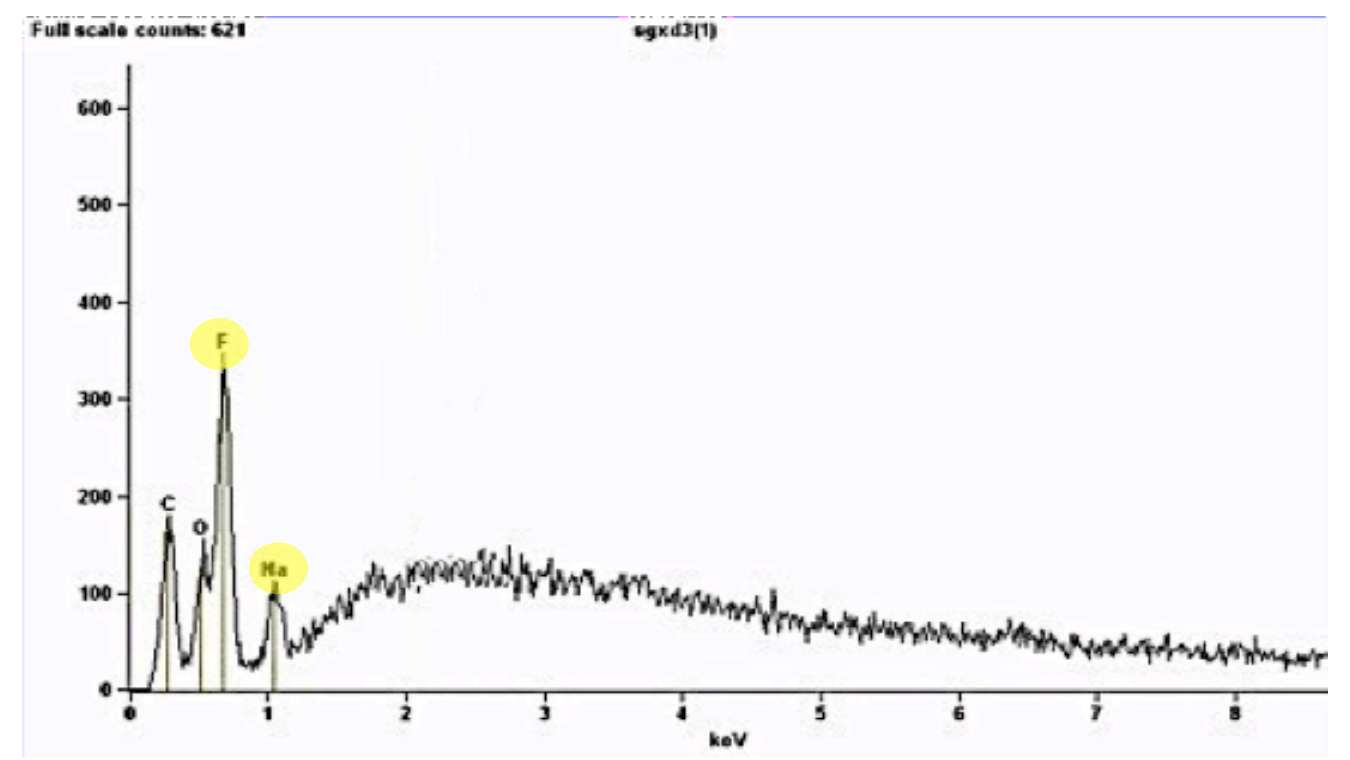

Figure 6 Typical XRD SEM spectrum of defect 


\begin{tabular}{|l|l|l|}
\hline \multicolumn{1}{|c|}{ Sample } & F & Na \\
\hline 1 & 37,98 & 16,69 \\
\hline 2 & 42,77 & 17,7 \\
\hline 3 & 42,58 & 16,25 \\
\hline 4 & 50,45 & 17,68 \\
\hline 5 & 43,31 & 16,32 \\
\hline 6 & 41,07 & 24,24 \\
\hline 7 & 45,16 & 12,07 \\
\hline 8 & 39,90 & 17,96 \\
\hline 9 & 46,53 & 16,73 \\
\hline 10 & 41,67 & 14,24 \\
\hline 11 & 37,23 & 15,12 \\
\hline 12 & 40,67 & 17,70 \\
\hline 13 & 47,00 & 16,25 \\
\hline 14 & 49,99 & 17,63 \\
\hline 15 & 40,01 & 15,43 \\
\hline 16 & 39,87 & 19,33 \\
\hline 17 & 40,93 & 17,77 \\
\hline 18 & 39,45 & 17,96 \\
\hline 19 & 43,21 & 16,73 \\
\hline 20 & 41,67 & 14,24 \\
\hline & & \\
\hline
\end{tabular}

\section{Fluorine}

$(42 \pm 1) \%$

\section{Sodium}

$(16,9 \pm 0,8) \%$

Up to $100 \%$

\section{Other Elements $(\mathrm{S}, \mathrm{O}, \mathrm{H})$}




\section{A NaF defects generation mechanism}

The gap is operated at $9 \mathrm{kV}$ high voltage supply. When the voltage is applied, a ion motion occurs both in the bakelite and in the linseed oil. The ion motion brings contaminants (contained inside the material) onto the surface layer. In particular, we propose that the $\mathrm{NaF}$ observed be due to a two-phase process.

\subsection{First phase}

\subsubsection{First phase (a)}

The electric current crosses the polymers because of movements of free ions inside the polymer lattice. Sodium is one of these ions that, when high-voltage is applied, will move towards the cathode as $\mathrm{Na}^{+}$. The motion of $\mathrm{Na}^{+}$(an excellent electrolyte) towards the cathode is facilitated by the presence of water inside bakelite. Simultaneously, the fat acids hydrolysis occur inside the linseed oil

$$
\mathrm{R}-\mathrm{COOH} \rightarrow \mathrm{R}-\mathrm{COO}-+\mathrm{H}^{+}
$$

where $\mathrm{R}$ stand for a long hydrocarbon chain (C11-C17). The hydrogen ions also move to the cathode.

Gamma radiations, $\mathrm{O}_{2}, \mathrm{iC}_{4} \mathrm{H}_{10}$ and every organic solvent catalyze the reaction 1.1.

When R-COO- and $\mathrm{Na}^{+}$are in contact, the following reaction occurs

$$
\mathrm{R}-\mathrm{COO}-+\mathrm{Na}^{+} \rightarrow \mathrm{R}-\mathrm{COONa}
$$

and a metallic soap 2 is formed close to the bakelite inner surface.

\footnotetext{
${ }^{2}$ The reaction is similar to the one that occurs in the oil painting canvas. Soaps or surfactants are fat acid salts. They are made of a hydrophilic head and a lipophilic long hydrocarbon chain.
} 
In fig. 7 the first phase is schematized.

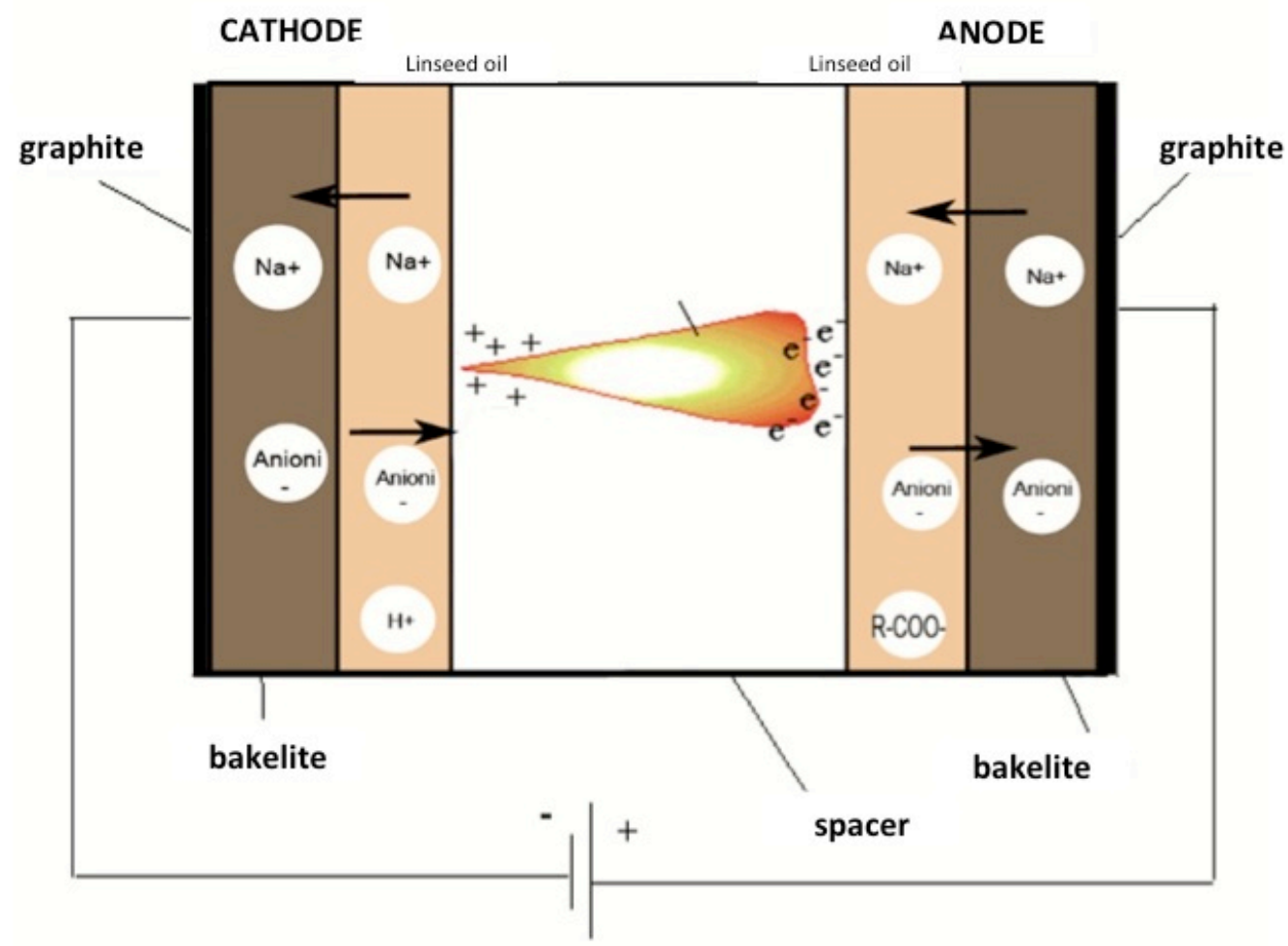

Figure 7 Migration of ions in gap under electric field 


\section{First phase (b)}

Some areas of the oil layer can be not perfect, there are higher oil thickness, or surface cracks; here electrical discharges can occur. In fact a $9000 \mathrm{~V}$ voltage applied to the gap create an electrical field of 50-80 kV/m on the top of a generic linseed oil drop (Fig.3) .

When the ions generated from the linseed oil and the bakelite move to such areas when an electrical discharge occurs, the $\mathrm{SF}_{6}$ splits in ions.

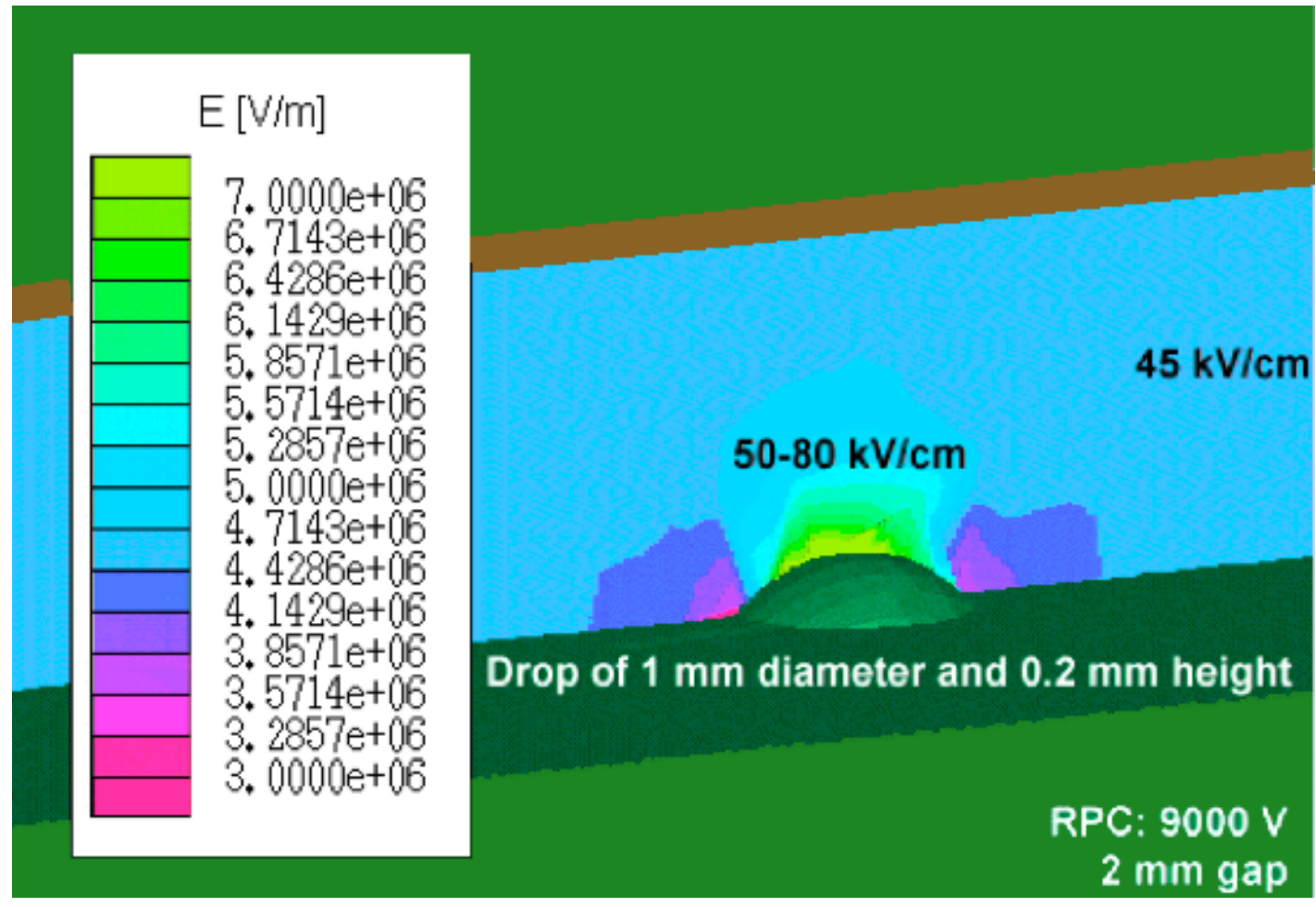

Figure 8 bla bla from poster

This happens in every common industrial switch at medium and high voltage, where $\mathrm{SF}_{6}$ stops the electric arc that each closing or opening of the circuit create; in these switches, without contaminants, $\mathrm{SF}_{6}$ will form again soon after its breaking. 
Some of the $\mathrm{SF}_{6}$ decomposition products are $\mathrm{SF}_{4}, \mathrm{SOF}_{2}, \mathrm{SO}_{2}, \mathrm{SO}_{2} \mathrm{~F}_{2}, \mathrm{SOF}_{4}, \mathrm{HF}$, all are very reactive. In the gaps, where there are other molecules, these reactions take place:

$\mathrm{SF}_{4}+\mathrm{H}_{2} \mathrm{O} \rightarrow \mathrm{SOF}_{4}+\mathrm{HF}$

$\mathrm{SOF}_{4}+\mathrm{H}_{2} \mathrm{O} \rightarrow \mathrm{SO}_{2}+\mathrm{HF}$

so that we find mainly $\mathrm{HF}$ and $\mathrm{SO}_{2}$ on the surface. The presence of a large amount of water, in fact, move to right the equilibrium.

HF is formed from the Freon decomposition too.

\subsection{Second phase}

HF react with the metallic soap according to the reaction:

$\mathrm{R}-\mathrm{COONa}+\mathrm{HF} \rightarrow \mathrm{R}-\mathrm{COOH}+\mathrm{NaF}$

where R-COONa is a salt (from a weak acid and a strong base) and HF is a weak acid. The products are a fat acid and sodium fluoride; the latter forms between the oil surface and the bakelite.

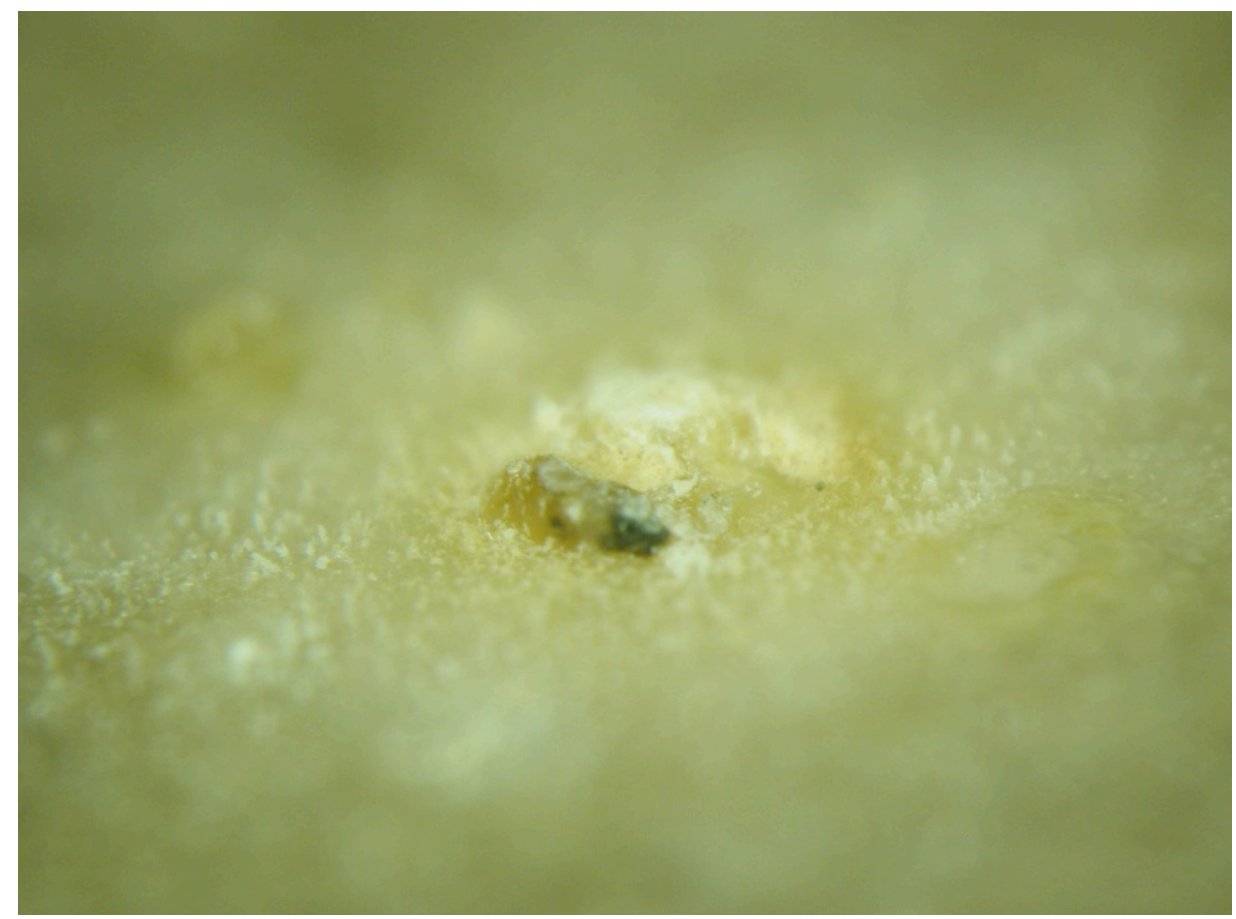

Figure 9 3D reconstruction of $\mathrm{NaF}$ on the bakelite surface. 
The reactions 1.3, 1.4 and 1.5 are catalyzed by gamma-ray radiation, which helps to overcome the kinetic barrier.

\section{Conclusions and outlook}

A model for the chemical formation of defects is proposed for the first time. The distribution of defects clusters on the gas inlet and gas outlet areas, a Computer Fluid Dynamic analysis is in progress. The high-statistics SEM/XRD analysis of defects confirms the presence of $\mathrm{Na}$ in defected samples only. Being $\mathrm{Na}$ an excellent electrolyte, its diffusion in bakelite is facilitated by the humidity contained.

\section{References}

[1] R. Santonico, R. Cardarelli (Rome U.) Development Of Resistive Plate Counters. Nucl. Instr. Meth. 187 (1981) 377-380

[2] R. de Asmundis, Application of Gas Chromatographic analysis to RPC detectors in the ATLAS experiment at CERN-LHC JINST 2, T06001 (2007)

[3] R. de Asmundis, Online gas analysis and diagnosis for RPC detectors in the ATLAS experiment Nucl. Instrum. Meth. A 572, 40 (2007)

[4] J. Va'vra, "Physics and chemistry of aging: Early developments," ICFA Instrum. Bull. 24, 1 (2002) Nucl. Instr. Meth. A 515, 1 (2003)

[5] J. Va'vra, "Summary of session 6: Aging effects in RPC detectors," Nucl. Instr. Meth. A $\mathbf{5 1 5}, 354$ (2003)

[6] G. Aielli et al., Fluoride production in RPCs operated with F-compound gases,. 2006. 6pp. Prepared for 8th Workshop on Resistive Plate Chambers and Related Detectors, Seoul, Korea, 10-12 Oct 2005. Published in Nucl. Phys. Proc. Suppl. 158: 143-148, 2006

[7] M. Abbrescia et al., "Gas analysis and monitoring systems for the RPC detector of CMS at LHC," LNF-06-34-P, LNF-04-25-P, Jan 2007. 9pp. Presented by S. Bianco on behalf of the CMS RPC Collaboration at the 2006 IEEE Nuclear Science Symposium (NSS), Medical Imaging Conference (MIC) and 15th International Room Temperature Semiconductor Detector Workshop, San Diego, California, 29 Oct - 4 Nov 2006. arXiv:physics/0701014

[8] M. Abbrescia et al., Proposal for a Systematic Study of the CERN Closed Loop Gas System Used by the RPC Muon Detectors in CMS, Frascati preprint LNF-06/27(IR), available at http://www.lnf.infn.it/sis/preprint/

[9] S. Bianco et al., Chemical Analyses Of Materials Used In The Cms Rpc Muon Detector CMS-NOTE-2010-006.

[10] S. Chatrchyan et al. [CMS Collaboration], "The CMS experiment at the CERN LHC," JINST 3 (2008) S08004

[11] M. Abbrescia et al., "HF Production In Cms-Resistive Plate Chambers," Nucl. Phys. Proc. Suppl. 158 (2006) 30. NUPHZ, 158, 30 
[12] M. Abbrescia et al., "Study Of Long-Term Performance Of Cms Rpc Under Irradiation At The Cern Gif,"Nucl. Instrum. Meth. A 533, 102 (2004)

[13] S. Agosteo et al., Nucl. Instrum. Meth. A 452 (2000) 94

[14] S. Altieri et al., Nucl. Instr. Meth. in Physics Research A 456 (2000) 132$\} 136$

[15] CMS RPC bakelite sheets are produced by PANPLA, Pavia, Italy.

[16] The linseed oil used for CMS RPC is produced by GALLO SRL, 61030 Tavernelle di Serrungarina, Italy.

[17] C.Pucci, MD Thesis, Sapienza Universita` di Roma (2006) (in Italian). Frascati preprint LNF-06/31(Thesis). 